\title{
Avaliação do atraso de pacotes em filas para tráfego estocástico em rajada no simulador de redes com base no modelo fluido
}

\author{
Rudolfo E. Rüncos e Eduardo P. Ribeiro
}

\begin{abstract}
Resumo-Este trabalho avalia através de simulações no network simulator (ns2) as condições em que o atraso em fila de pacotes de um tráfego estocástico tipo rajada (modelo exponencial on/off ) pode ser calculado com boa aproximação por um simples modelo fluido. Tanto os parâmetros do tráfego (tempo relativo de rajada e ociosidade) quanto a fila inicial são variados e pode-se observar quando a representação pelo modelo fluido não é adequada.
\end{abstract}

Palavras-Chave-modelo fluido, tráfego on/off, atraso, fila.

Abstract-This article evaluates through simulations on network simulator (ns2) the conditions where queue packet delays due to an bursty traffic (exponetial on/off) may be calculated with good approximation using a simple fluid model. Both traffic parameters (relative burst/idle times) and initial queue are tested and adequacy of fluid model representation can be verified.

Keywords - fluid model, on/off traffic, delay, queue.

\section{INTRODUÇÃO}

A teoria de filas em muito tem contribuido para o estudo e dimensionamento das redes de comunicações de dados de uma maneira geral. O tempo médio de espera em fila pode ser usualmente determinado supondo-se o processo de chegada de pacotes marcoviano e um estado estacionário de operação onde a utilização média do sistema seja abaixo de $100 \%$. No entanto situações momentâneas quando se deseja calcular o atraso médio após um certo período de operação em que a taxa média de entrada é maior que a taxa de saída não são contempladas por esta teoria. Tais situações são de interesse na avaliação de protocolos que envolvam operação de tempo real como, por exemplo, comunicações de áudio e vídeo ou comunicações de controle de processos industriais. Uma abordagem simples para essas situações consiste na aproximação do tráfego estocástico por um modelo de fluxo de fluido. Esta aproximação geralmente é razoável quando o sistema se encontra em condição de tráfego pesado com filas longas e ocupadas. Kleinrock [1] comenta justamente a importância da análise destas situações críticas visto que a situação de filas vazias não apresentam sério problema para a o desempenho dos sistemas. Recentemente este tipo de modelagem por fluxo fluido tem atraido a atenção de pesquisadores para acelerar o processo de simulação principalmente como uma solução escalável para grandes redes [2][3]. Este trabalho visa fazer uma investigação inicial da validade do modelo fluido simples

Rudolfo Runcos e Eduardo Parente Ribeiro, Departamento de Engenharia Elétrica, Universidade Federal do Paraná, Curitiba, PR, Brasil, E-mails: rrscar@gmail.com, edu@eletrica.ufpr.br. para simulações realizadas no NS2 - Network Simulator com tráfego estocástico em rajada (tipo on/off). Objetiva-se determinar o tempo de espera em fila (ou de forma equivalente o tamanho da fila) após um período de operação no qual a taxa média do tráfego estocástico de entrada é superior à taxa de saída da fila. Devido a natureza aleatória do processo, várias simulações são realizadas sendo estabelecido o intervalo de confiança $(95 \%)$ dos resultados obtidos.

\section{Metodologia}

A topologia considerada para teste consiste na fila com capacidade de saída de 1Mbps. O protocolo de transporte utilizado é o UDP e todos os pacotes apresentam um tamanho de 1000 bytes. No início de cada simulação foi usado um tráfego CBR para preencher a fila e evitar que ela fique vazia durante a simulação.

Quando o tempo de simulação é $t=1$ s o tráfego CBR é desligado e o tráfego exponencial on/off tem início. Os parametros do tráfego on/off são: tempo médio ocioso - idle time $\left(T_{\text {off }}\right)$, tempo médio de rajada - burst time $\left(T_{o n}\right)$ e taxa de rajada - burst rate $\left(R_{\text {burst }}\right)$. Estes parâmetros foram escolhidos de forma a sempre se ter uma taxa média $R_{i n}=1,1 \mathrm{Mbps}$. Por simplicidade foi fixado $T_{o n}=50 \mathrm{~ms}$ para todas as simulações. A eq. (1) mostra o calculo da taxa média de entrada de acordo com o modelo de fluxo fluido:

$$
R_{\text {in }}=\frac{R_{\text {burst }} T_{\text {on }}}{T_{\text {on }}+T_{\text {off }}}
$$

O tempo durante o qual o tráfego on/off permaneceu ligado foi escolhido como 10s. Deseja-se estudar o comportamento médio do atraso durante o tempo de simulação mas descontando-se o atraso inicial. Assim o atraso (A) é medido como a diferença entre o tempo de entrada e saída da fila do último pacote da simulação (mais proximo de 10s) menos o atraso inicial $\left(A_{0}\right)$. $\mathrm{O}$ valor do atraso pelo modelo fluido é dado por $A=\left[\left(R_{\text {in }}-R_{\text {out }}\right) T_{\text {sim }}\right] / R_{\text {out }}$ e este é sempre igual a 1s para todas simulações. Ou seja, de acordo com o modelo fluído, as simulações deveriam chegar num atraso médio de $1 \mathrm{s.}$

O primeiro caso simulado foi para $A_{0}=15 \mathrm{~s}$. Este caso garante a fila sempre cheia durante o tempo de simulação, servindo, portanto, como caso de referência para os demais. Dentro deste caso, valores de $T_{\text {off }}$ foram considerados e o respectivo valor de $R_{\text {burst }}$. A tabela I mostra os valores adotados para $T_{o f f}$ e $R_{\text {burst }}$ em cada situação, já que $T_{o n}=50 \mathrm{~ms}$ e 
$R_{\text {in }}=1,1 M b p s$ são mantidos fixos. Como o desvio padrão do atraso $\left(\sigma_{A}\right)$ aumenta com o aumento de $T_{\text {off }}$ (e $\left.R_{\text {burst }}\right)$, decidiu-se manter um intervalo de confiança de $95 \%$ com cerca de 0,1 s de largura. $\mathrm{O}$ número de simulações realizadas para alcançar esse objetivo em cada situação também se encontra na tabela. Os outros casos estudados foram para $A_{0}=6 \mathrm{~s}$, $A_{0}=3 s, A_{0}=1 s$ e $A_{0}=0 s$. Nenhum destes casos garante fila cheia ao longo de toda a simulação. Para estes casos, foram simuladas apenas 3 das situações: $T_{\text {off }}=5 \mathrm{~ms}, T_{\text {off }}=50 \mathrm{~ms}$ e $T_{\text {off }}=150 \mathrm{~ms}$.

TABELA I

VALORES USADOS NAS SIMULAÇÕES

\begin{tabular}{|c|c|c|c|c|}
\hline$T_{\text {on }}(\mathrm{ms})$ & $T_{\text {off }}(\mathrm{ms})$ & $R_{\text {burst }}$ Mbps & $\sigma_{A}(\mathrm{~s})$ & $\mathrm{n}$ \\
\hline \hline 50 & 150 & 4,4 & 1,682 & 4200 \\
\hline 50 & 100 & 3,3 & 1,258 & 2400 \\
\hline 50 & 75 & 2,75 & 1,030 & 1800 \\
\hline 50 & 50 & 2,2 & 0,775 & 900 \\
\hline 50 & 25 & 1,65 & 0,448 & 315 \\
\hline 50 & 5 & 1,21 & 0,098 & 20 \\
\hline 50 & 2,5 & 1,155 & 0,054 & 7 \\
\hline 50 & 0,25 & 1,1055 & 0,004 & 3 \\
\hline
\end{tabular}

\section{RESULTADOS}

A figura 1 mostra a média dos atrasos obtidos nas simulações para cada situação no caso de $A_{0}=15 \mathrm{~s}$ (fila sempre cheia). Neste gráfico, o eixo das abscissas corresponde à razão $T_{o n} / T_{\text {off }}$. Assim, quanto mais à direita um ponto estiver no gráfico, menor o tempo de ociosidade e menor a variabilidade, aproximando-se no limite a um tráfego CBR. Quanto mais à esquerda, mais os tempos ociosos aumentam e maior é a taxa de transmissão em momentos de rajada, o que faz com que a variabilidade do tráfego aumente e, por conseguinte, também aumente a variância do atraso. $\mathrm{O}$ gráfico mostra também os intervalos de confiança de $95 \%$ para cada situação onde o número de simulações foi propositadamente variado conforme tabela I para que cada intervalo de confiança ficasse próximo de $0,1 \mathrm{~s}$. Observa-se que nesta situação de fila cheia, os intervalos de confiança sempre contém o valor previsto teoricamente pelo modelo fluido, mesmo para situações de alta variabilidade do tráfego $\left(T_{o n} / T_{o f f}=1 / 3\right)$.

A figura 2 resume o resultado dos demais casos simulados. Os resultados das situações com fila inicial menor mostram que para situações de grande variabilidade do tráfego, a média deste se desvia da esperada pelo modelo fluido. Esse desvio é sempre no sentido de aumentar o atraso médio. Quanto menos cheia estiver a fila, maior se torna o aumento do atraso. $\mathrm{O}$ motivo que leva ao aumento do atraso médio é que durante o período em que a fila fica vazia, não há escoamento de tráfego. Dessa forma, numa situação onde a fila fica por algum tempo vazia, a taxa média de saída da fila é menor do que a taxa do enlace. Como a taxa de entrada média $R_{i n}$ se mantém a mesma, pois independe do estado da fila, o atraso na fila tende a ser maior nessas situações. Portanto, em situações em que não se pode garantir uma fila sempre cheia, o modelo fluído se afasta de uma boa representação. Porém, em tipos de tráfego de baixa variabilidade, mesmo nos momentos de ociosidade a fila não consegue ser completamente esvaziada, ou não fica vazia por muito tempo. Nestes casos o modelo fluido é uma boa aproximação para o tempo médio de espera em fila e os resultados obtidos no simulador corroboram isto.

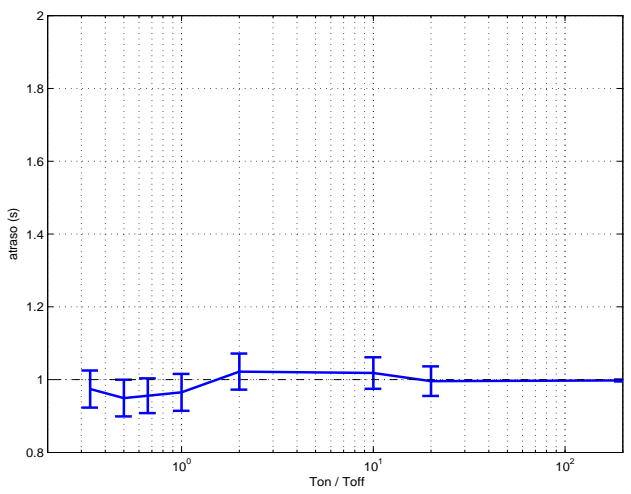

Fig. 1. Simulação com fila cheia. Atraso previsto de 1s

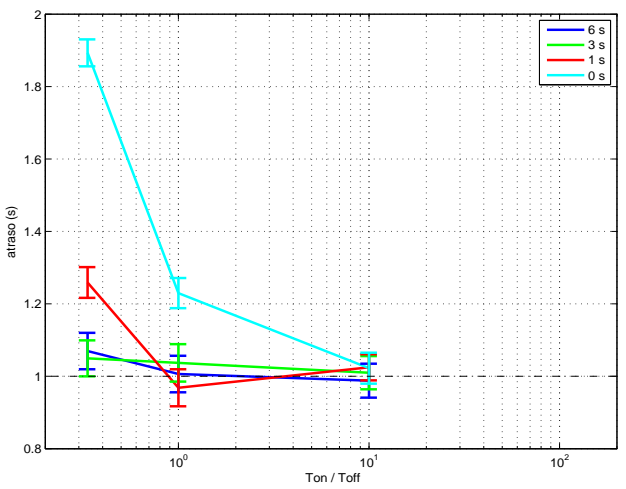

Fig. 2. Simulação com fila parcialmente cheia. Atraso previsto de 1s

\section{CONCLUSÕES}

Uma grande quantidade de simulações foi realizada no simulador de redes (ns2) para se verificar o atraso em fila durante um período de operação onde a taxa média de entrada do tráfego exponencial on/off era maior que a taxa de saída. Calculou-se o intervalo de confiança (95\%) para se avaliar quando que as simulações correspondiam ao valor previsto teoricamente segundo um modelo fluido. Verificou-se que quando tráfego apresenta períodos de ociosidade pequenos (baixa variabilidade) a fila inicial pode estar vazia que os os breves períodos de ociosidade não vão afetar a aproximação do modelo fluido. Porém, para os casos de tráfego com grandes períodos de ociosidade (razão $T_{o n} / T_{o f f}$ baixa), se a fila inicial não tiver tamanho suficientemente grande isto implicará numa subestimação do atraso pelo modelo fluido.

\section{REFERÊNCIAS}

[1] L. Kleinrock, Queueing Systems, v.II. John Wiley \& Sons, 1976.

[2] B. Liu, D. R. Figueiredo, J. K. Yang Guo e D. Towsley, A study of networks simulation efficiency: Fluid simulation vs. packet-level simulation. In Proc. of the IEEE INFOCOM, volume 3, pages 12441253, Apr. 2001.

[3] Y. Liu, F. L. Presti, V. Misra, D. Towsley e Y. Gu, Fluid models and solutions for large-scale ip networks. In Proceedings of ACM SIGMETRICS'03. San Diego, CA. 2003. 